\section{Oxygen: Implications for Wound Healing}

\author{
Diego M. Castilla, Zhao-Jun Liu, ${ }^{1,2}$ and Omaida C. Velazquez ${ }^{1,2, *}$ \\ ${ }^{1}$ Division of Vascular Surgery, DeWitt Daughtry Family Department of Surgery, Miller School of Medicine; \\ ${ }^{2}$ Sylvester Comprehensive Cancer Center; University of Miami, Miami, Florida.
}

Background: Oxygen is vital for healing wounds. It is intricately involved in numerous biological processes including cell proliferation, angiogenesis, and protein synthesis, which are required for restoration of tissue function and integrity. Adequate wound tissue oxygenation can trigger healing responses and favorably influence the outcomes of other treatment modalities.

The Problem: Chronic ischemic wounds fail to heal appropriately secondary to extreme hypoxia that leads to cellular demise. Wound tissue hypoxia is typically greater at the center of the wound. Accordingly, oxygen requirements of the regenerating tissue will vary.

Basic/Clinical Science Advances: As oxygen levels decrease within the wound, cell response mechanisms (hypoxia inducible factor [HIF]) trigger the transcription of genes that promote cell survival and angiogenesis. HIF stabilizers are currently being tested to determine wound healing potential. Clinically, topical oxygen therapy (TOT) has been proved as an effective therapeutic modality for chronic wounds. TOT is reputed to have several advantages over hyperbaric oxygen therapy. Namely, TOT has a lower risk of oxygen toxicity, it is less expensive and is relatively easy to apply to target areas.

Clinical Care Relevance: Wound tissue oxygen is necessary for appropriate wound healing; however, the relative complexity of the healing process requires a multifaceted approach for successful healing outcomes. A key component of this multifaceted approach should be specific oxygen dosing as a function of tissue hypoxia.

Conclusion: New treatment approaches that exploit cell hypoxia sensing and response mechanisms and that enable the precise application of oxygen therapy to hypoxic areas of regenerating tissue are very promising.

\section{BACKGROUND}

WOUND HEALING Is a dynamic and carefully orchestrated process involving blood cells, cytokines, parenchymal cells (i.e., fibroblasts), and extracellular matrix reorganization. In the normal wound, this interactive process ultimately restores dermal and epidermal functional integrity in a sequential and timely fashion.

A key moderator of normal wound healing is oxygen. Indeed, oxygen is a requirement for various processes in the healing of wounds including collagen deposition, epithiliazation, fibroplasia, angiogenesis, and resistance to infection. A limitation in the delivery of $\mathrm{O}_{2}$-richblood to the wound logic healing.

The limitation of oxygen delivery to the wound is often multi-factorial; however, the end result is a hypoxic (oxygen deprived) wound microenvironment characterized by insufficient nutrient and oxygen delivery to the regenerating tissue. In this context, the state-of-wound tissue tissue, therefore, impedes physio-

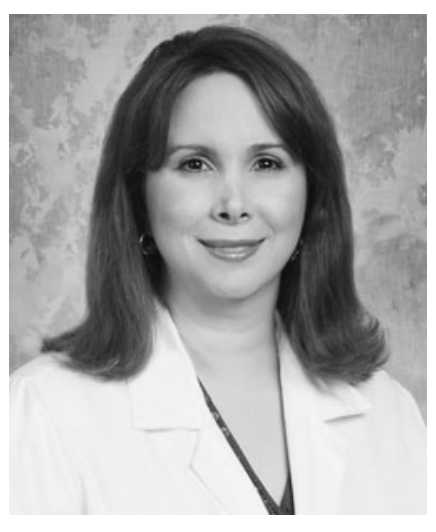

Omaida C. Velazquez

Submitted for publication July 29, 2011

*Correspondence: Division of Vascular and Endovascular Surgery, DeWitt Daughtry Family Department of Surgery, University of Miami Miller School of Medicine, Room 3016 Holtz Center, JMH East Tower, 1611 N.W. 12th Ave., Miami, FL (e-mail: ovelazquez@med.miami.edu).
Abbreviations and Acronyms
BMeNOS = bone marrow endothelial nitric oxide synthase
$\mathrm{EC}=$ endothelial cell
EPCs $=$ endothelial progenitor cells
HBOT = hyperbaric oxygen therapy
$\mathrm{HIF}=$ hypoxia inducible factor
$\mathrm{NO}=$ nitric oxide
NOS $=$ nitric oxide synthase
NOX $=$ NADPH oxidase
PHDs = prolyl hydroxylase domain enzymes
ROS $=$ reactive oxygen species
TOT = topical oxygen therapy
VEGF = vascular endothelial growth factor 
oxygenation is a major determinant of healing outcomes. $^{1,2}$

Tissue confronted with an acute, mild-tomoderate hypoxic challenge typically adapts. Conversely, tissue confronted with chronic, severe hypoxia does not survive. ${ }^{1}$ The same holds true for elevated wound tissue oxygen partial pressures where a moderate hyperoxic challenge can stimulate the production of growth factors and the formation of new blood vessels, whereas extreme hyperoxia can induce mitochondrial apoptosis, growth arrest, and oxidative stress via the formation of reactive oxygen species (ROS). Thus, the inherent complexity of the healing process mandates the precise combination of hypoxic cellular signaling and antioxidant defense mechanisms with adequate tissue oxygenation. ${ }^{1}$

\section{TARGET ARTICLE}

Sen CK. Wound healing essentials: let there be oxygen. Wound Repair Regen 2009; 17: 1.

\section{CLINICAL PROBLEM ADDRESSED}

Chronic skin wounds are wounds that have failed to proceed through an orderly and timely reparative process to produce anatomic and functional integrity. Approximately 7 million patients are affected by chronic wounds in the United States alone, and an estimated $\$ 25$ billion dollars is spent annually on the treatment of such wounds. Unfortunately, these numbers continue to increase as a result of an aging patient population and the increased prevalence of diabetes, obesity, and atherosclerosis worldwide. ${ }^{3}$ To this end, concentrated scientific efforts have continued to focus on the biological mechanisms that underlie wound complications with the ultimate goal of finding the most effective therapeutic modalities for afflicted patients.

The major role oxygen plays in many of the essential wound healing processes has also attracted considerable clinical interest, and investigations addressing the treatment of wound tissue hypoxia have yielded compelling data. ${ }^{4-6}$ In addition, scientific studies targeting the oxygen response signaling pathways within the wound tissue milieu are continuing to enhance our understanding of the biological and chemical pathways involved in the hypoxia sensing and response systems. This is of particular importance for the exploitation of new treatments that target hypoxia-response mechanisms within the healing tissue, thus making them useful in the wound clinic when combined with appropriate tissue oxygenation.

\section{RELEVANT BASIC SCIENCE CONTEXT}

In the chronic wound microenvironment, there inevitably exists a substantial imbalance between the supply of oxygen and the high energy demand of the healing tissue. From a molecular standpoint, the key factors that propagate this imbalance include the following: (1) the increased utilization of oxygen by the hypermetabolic regenerating tissue, (2) the sustained and increased production of ROS by phagocytes (respiratory burst), and (3) reduction-oxidation (redox) signaling. ${ }^{1}$

Redox signaling and ROS generation is a process reliant on the NADPH oxidase (NOX) family of enzymes. The redox signals produced serve critical roles within the wound bed: (1) they provide the phagocytic immune cells with potent disinfecting capabilities, (2) in physiologic concentrations, they function as signaling molecules that mediate various responses, including cell proliferation, migration, differentiation, gene expression, and vascular tone. ${ }^{7,8}$

Although NOX-dependent $\mathrm{O}_{2}$ consumption is vital for appropriate redox signaling within the wound, it is important to understand that the optimal production of redox signals is imperative, as excessive ROS production can rapidly contribute to a hypoxic wound environment and cell death. Accordingly, the severely hypoxic wound environment will lack the essential $\mathrm{O}_{2}$ necessary for crucial redox signaling and appropriate wound healing. ${ }^{1}$ To mitigate the effects of the hypoxic environment, many cells use the hypoxia inducible factor (HIF) cascade, which activates multiple genes involved in cell proliferation, angiogenesis, and glucose metabolism.

HIF-1 is a protein composed of two subunits: HIF- $1 \alpha$ and HIF- $1 \beta$. The latter is constitutively expressed within the cell, whereas the former contains the- $\alpha$ subunit tightly regulated by $\mathrm{O}_{2}$ dependent enzymes referred to as prolyl hydroxylase domain enzymes (PHDs). Under normoxic (oxygen replete) conditions, the PHDs inactivate the HIF$1 \alpha$ subunit. Conversely, hypoxic conditions inactivate the PHDs; stabilization of the HIF- $1 \alpha$ subunit ensues, thus allowing for the formation of the HIF$1 \alpha /$ HIF- $1 \beta$ protein complex, which then translocates into the nucleus. The HIF protein complex can then bind to hypoxia responsive elements within the promoter regions of various genes that are turned on in a hypoxic environment. ${ }^{9}$

\section{EXPERIMENTAL MODEL OR MATERIAL: ADVANTAGES AND LIMITATIONS}

There have been extensive basic science and clinical studies investigating the pathophysiology 
of wound healing. Basic science research has utilized several animal models of acute wounding to understand the underlying principles and mechanisms involved in the healing of acute wounds, ischemic wounds, and hypoxic wounds. Nonetheless, it is apparent that principles demonstrated in acute wounds can apply to chronic wounds as well.

Acute wounds provide a very precise model that can be standardized. This permits the precise manipulation of certain experimental parameters, which facilitates the investigation of specific biochemical pathways and cell interactions within the healing wound. In addition, the outcome of acute wounds can be studied over a relatively short period of time. Thus, data can be gathered with relative efficiency.

Chronic wounds, on the other hand, typically vary in size, duration, location, and etiology. They are difficult to reproduce in the laboratory, which makes standardization problematic. Moreover, chronic wounds take at least several months to heal, thus making data collection more protracted. For this reason, studies dealing with chronic wounds are typically outcome studies that utilize systemic oxygen therapy, TOT, and wound oxygen measurement modalities (e.g., HBOT, TOT, transcutaneous oximetry, electronic paramagnetic resonance oximetry), to investigate patient outcomes.

\section{DISCUSSION OF FINDINGS AND RELEVANT LITERATURE}

Wound healing is an angiogenesis dependent process reliant on blood vessels to deliver nutrients and oxygen to the regenerating tissue. The formation of new blood vessels (neovascularization) in the wound proceeds in two ways under the influence of angiogenic stimuli, such as vascular endothelial growth factor (VEGF) - the most potent factor for neovascularization: (1) angiogenesis, the process of new vessel sprouting from existing vessels and residing endothelial cells (ECs); (2) vasculogenesis, the recruitment and differentiation of circulating endothelial progenitor cells (EPCs) to form new vessels. ${ }^{5,10}$

Accordingly, the efficacy of oxygen therapy in the healing wound is, in part, mediated by the enhancement of the angiogenic response. Numerous in vivo studies have demonstrated increased VEGF expression in wounds treated with systemic oxygen therapy (HBOT), and in vitro studies have shown increased levels of VEGF mRNA in cultured cells involved in the healing process. ${ }^{5,11,12}$ Interestingly, the increased VEGF expression in wounds treated with HBOT appears to be mediated, in part, by the stabilization of HIF, which subsequently promotes the transcription of the VEGF gene. ${ }^{10,13}$ It is important to note that there have been conflicting data with regard to HBOT, HIF, and wound healing. HBOT may affect HIF-1 expression depending on tissue type and the type of wound; that is, HIF-1 levels may differ in the acute healing phase compared with the late healing phase, or in ischemic wound healing. ${ }^{10,14}$

In addition to the localized induction of angiogenesis, HBOT has been shown to stimulate vasculogenesis by enhancing the production of bone marrow endothelial nitric oxide synthase (BMeNOS); BMeNOS produces nitric oxide (NO). NO subsequently enhances the mobilization of EPCs, which home to the wound and contribute to the formation of neovessels. ${ }^{15}$ Further, HBOT is involved in fibroblast activation, the up-regulation of growth factors, the reduction of the inflammatory response, and wound disinfection. ${ }^{6}$ It is these data that that lend support to the utilization of HBOT as an adjunctive care measure for diabetic lower extremity ulcers. ${ }^{16}$

HBOT remains a well-established, adjunctive treatment for diabetic lower extremity wounds refractory to standard care practices. The majority of the recent clinical outcome studies continue to provide strong and convincing evidence that adjunctive HBOT, in the setting of diabetes, decreases the risk of major amputation and markedly improves wound healing outcomes. ${ }^{6,16}$ However, the effect of HBOT on chronic wounds associated with other pathologies (arterial ulcers, pressure ulcers, and venous ulcers) requires further rigorous randomized trials to appropriately validate outcomes. $^{6}$

Another promising therapy that enhances the oxygenation of cutaneous wounds is TOT. TOT is an $\mathrm{O}_{2}$ gas emulsion applied to the superficial wound tissue. Published data strongly suggest that TOT can generate a sustained increase in tissue oxygen levels; it can also enhance the angiogenic response in chronic human wounds by inducing VEGF production. ${ }^{5}$ Major advantages of TOT appear to be its independence of the wound microcirculation, its lower cost compared with systemic oxygen therapy, the lower risk of oxygen toxicity, and its relative simplicity of application and portability. Indeed, several clinical and animal studies have yielded promising results. ${ }^{4,17,18}$

The most recently reviewed is a prospective, controlled study that assessed diabetic foot ulcer healing outcomes in an outpatient setting. The 
TOT treatment group showed a significantly better healing outcome (85\%) compared with the control group (45\%) receiving standard wound care dressings. Improved outcomes were observed despite the larger, mean base-line wound area in the treatment group (4.1 vs. $\left.1.4 \mathrm{~cm}^{2}\right) .{ }^{4}$ Although the study was not randomized or double blinded, the significant differences in outcomes certainly contribute convincing evidence that TOT is an effective treatment option for problem wounds in which hypoxia of the superficial wound tissue impedes the healing process.

As previously outlined, HIF is an integral part of the cell hypoxia sensing and response mechanism that promotes cell survival via the induction of numerous genes. In the context of wound healing, HIF-1 transcriptional target products have been shown to enhance angiogenesis by regulating EC survival, migration and proliferation, vascular smooth muscle cell migration and proliferation, and by mobilizing circulating endothelial progenitor/stem cells to the periphery. HIF-1 has also been suggested to positively influence re-epithiliazation in the healing wound by increasing keratinocyte migration. Further contributions to wound healing have been documented in several diabetic and ischemic wound healing models, which have associated improvements in wound healing with sustained HIF-1 levels. ${ }^{19}$ Although numerous questions remain unanswered regarding specific molecular interactions, therapeutic agents that target HIF-1 appear promising.

\section{INNOVATION}

The heterogeneous redox environment of the healing wound presents varying tissue oxygen requirements. It can also be expected that more hypoxic wound tissue has greater concentrations of ROS secondary to cell death and the release of proinflammatory cytokines. As a result, the cellular antioxidant defense mechanisms are often overwhelmed. Recently, nanoparticles have shown promise in the area of wound healing with regard to the sustained delivery of antioxidants.

Fullerenes are carbon-based nanospheres with potent anti-inflammatory properties. In addition, fullerenes have large surface areas that make chemical and physical conjugation with small macromolecules relatively facile. In one recent study, several fullerene derivatives supported cell migration, induced wound closure in human skin explants, and greatly accelerated in vivo wound healing rates in mice. ${ }^{20}$ Administration of the fullerenes is systemic; therefore, biocompatibility, clearance, and toxicology are some of the many issues that need to be addressed in future studies for biomedical applications. In addition, distribution to ischemic wounds maybe limited as a result of decreased perfusion to these areas. However, nanotechnology remains an interesting and promising therapeutic option for hypoxic wounds.

\section{SUMMARY ILLUSTRATION}

The figure opposite demonstrates the key cellular processes mediated by oxygen and its derivatives during the process of wound healing. Oxygen is required for adenosine tri-phosphate synthesis within the mitochondria for oxidative metabolism; it is also essential for collagen synthesis and 


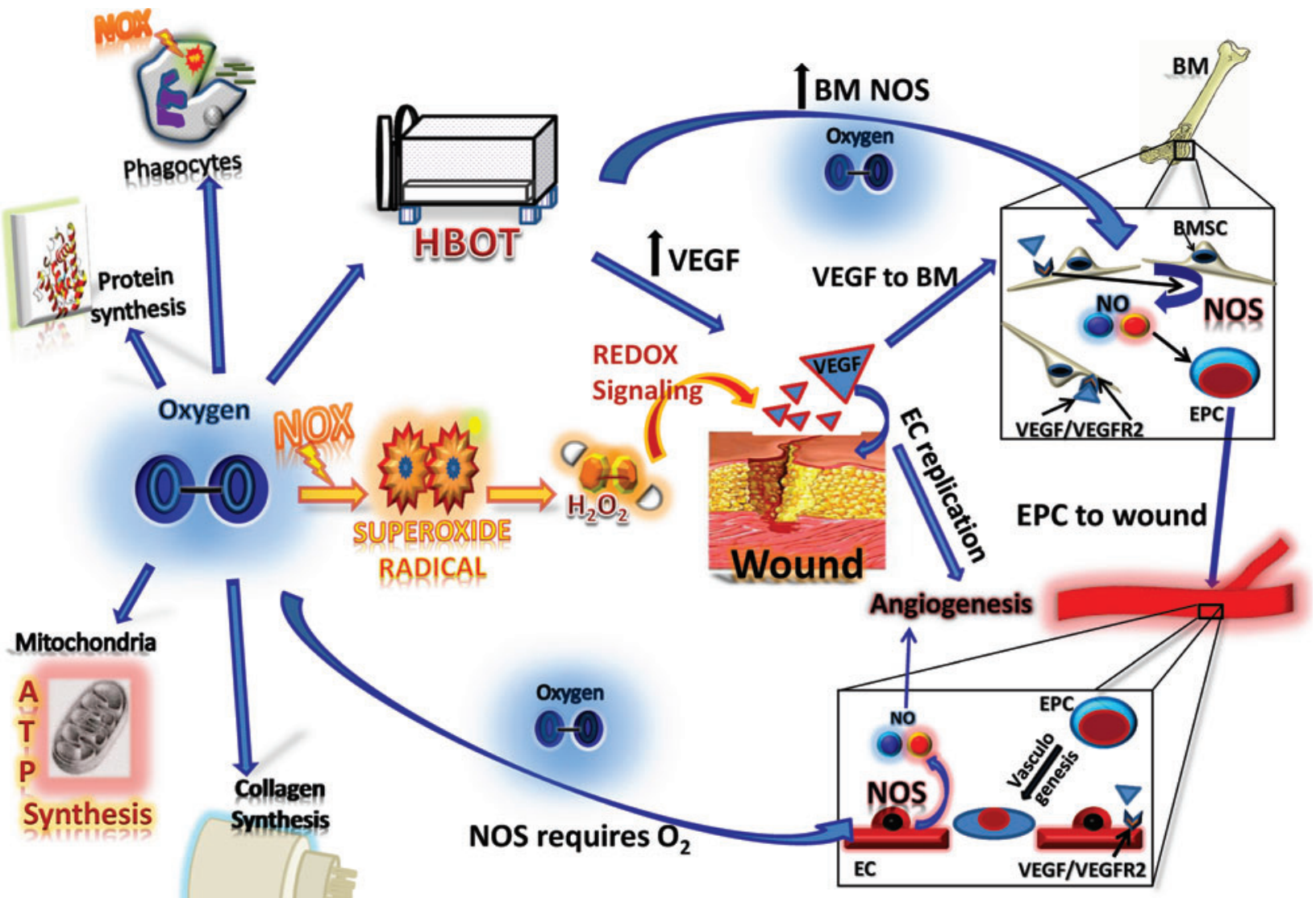

protein synthesis. Oxygen serves as a substrate for all NOX enzymes, which are a major source of ROS. The production of ROS during wound healing is critical for cell signaling, angiogenesis, and wound disinfection. The primary product of NOX catalysis is the superoxide anion.

Superoxide anion is the incomplete reduction byproduct of oxygen; specifically, it is oxygen with an additional, unpaired electron. Superoxide anion is the precursor for hydrogen peroxide, which has been shown to affect numerous key aspects of wound healing including cell proliferation, cell migration, and angiogenesis.

NO is produced by the nitric oxide synthase (NOS) family of enzymes, which require oxygen for the oxidation of the amino acid L-arginine to $\mathrm{NO}$ and citrulline. NO plays a critical role in the regulation of vascular tone and angiogenesis. Mobilization of EPCs from within the bone marrow is greatly enhanced by NO. Once mobilized from their bone marrow niche, EPCs home to the wound and incorporate into neovessels at ischemic sites. HBOT greatly enhances bone marrow NOS activity, thereby promoting the mobilization of EPCs from the bone marrow. Further, HBOT can in- crease levels of VEGF within the wound. VEGF is the central regulator of angiogenesis and vasculogenesis in the wound bed.

\section{CAUTION, CRITICAL REMARKS, AND RECOMMENDATIONS}

Systemic oxygen therapy (HBOT) and TOT have continued to demonstrate their efficacy for problem wounds refractory to standard care practices. As with any treatment, however, patient selection is of extreme importance to achieve success. If treatment goals are to augment the wound oxygen tension of the superficial tissue, then TOT should be expected to help. On the other hand, the systemic effects of HBOT are expected to raise systemic tissue $\mathrm{pO}_{2}$ to supraphysiological levels, which may assist in the treatment of concomitant problems (e.g., osteomyelitis). However, the elevation of systemic tissue oxygen levels makes oxygen toxicity a very real possibility. Although the majority of wound patients may not show overt signs of oxygen toxicity, clinicians prescribing HBOT should understand that the ability to handle oxidative stress is dependent on the expression of antioxidant proteins. Considering that many chronic wound 
patients are predisposed to malnutrition, which can result in a global reduction of protein synthesis, HBOT may not be suitable for some patients. ${ }^{1}$

\section{FUTURE DEVELOPMENT OF INTEREST}

Optimal wound oxygen therapy will require the precise application of oxygen treatment to hypoxic wound areas while sparing the adequately oxygenated wound tissue from the detrimental effects of hyperoxia. Technological advances in real-time oxygen sensing may soon enable the clinician to do so. However, it is vital that a multifaceted approach be undertaken, as oxygen therapy alone cannot remedy the multiple and complex limitations of the healing wound. Indeed, further studies to delineate these complex interactions will surely be necessary to identify and augment the key limitations of a given wound.

\section{ACKNOWLEDGMENTS AND FUNDING SOURCES}

This work was supported by grants from the National Institutes of Health (R01DK-071084 and R01GM081570).

\section{AUTHOR DISCLOSURE AND GHOSTWRITING}

The authors have no competing interests. The content of this article was expressly written by the authors listed. No ghostwriters were used to write this article.

\section{REFERENCES}

1. Sen CK: Wound healing essentials: let there be oxygen. Wound Repair Regen 2009; 17: 1.

2. Greif R, Akca O, Horn EP, Kurz A, Sessler DI, and Outcomes Research Group: Supplemental perioperative oxygen to reduce the incidence of surgicalwound infection. N Engl J Med 2000; 342: 161.

3. Sen CK, Gordillo GM, Roy S, Kirsner R, Lambert L, Hunt TK, Gottrup F, Gurtner GC, and Longaker MT: Human skin wounds: a major and snowballing threat to public health and the economy. Wound Repair Regen 2009; 17: 763.

4. Blackman E, Moore C, Hyatt J, Railton R, and Frye C: Topical wound oxygen therapy in the treatment of severe diabetic foot ulcers: a prospective controlled study. Ostomy Wound Manage 2010; 56: 24.

5. Gordillo GM and Sen CK: Evidence-based recommendations for the use of topical oxygen therapy in the treatment of lower extremity wounds. Int J Low Extrem Wounds 2009; 8: 105.

6. Kranke P, Bennett M, Roeckl-Wiedmann I, and Debus S: Hyperbaric oxygen therapy for chronic wounds. Cochrane Database Syst Rev 2004; 2: CD004123.

7. Chan EC, Jiang F, Peshavariya HM, and Dusting GJ: Regulation of cell proliferation by NADPH oxidase-mediated signaling: potential roles in tissue repair, regenerative medicine and tissue engineering. Pharmacol Ther 2009; 122: 97.
8. Weseler AR and Bast A: Oxidative stress and vascular function: implications for pharmacologic treatments. Curr Hypertens Rep 2010; 12: 154.

9. Fraisl P, Aragones J, and Carmeliet P: Inhibition of oxygen sensors as a therapeutic strategy for ischaemic and inflammatory disease. Nat Rev Drug Discov 2009; 8: 139.

10. Thom SR: Hyperbaric oxygen: its mechanisms and efficacy. Plast Reconstr Surg 2011; 127 (Suppl 1): $131 \mathrm{~S}$

11. Sheikh AY, Gibson JJ, Rollins MD, Hopf HW, Hussain Z, and Hunt TK: Effect of hyperoxia on vascular endothelial growth factor levels in a wound model. Arch Surg 2000; 135: 1293.

12. Staples KJ, Sotoodehnejadnematalahi F, Pearson $H$, Frankenberger $M$, Francescut $L$, ZieglerHeitbrock L, and Burke B: Monocyte-derived macrophages matured under prolonged hypoxia transcriptionally up-regulate HIF-1alpha mRNA. Immunobiology 2011; 216: 832.

13. Hunt TK, Aslam RS, Beckert S, Wagner S, Ghani QP, Hussain MZ, Roy S, and Sen CK: Aerobically derived lactate stimulates revascularization and tissue repair via redox mechanisms. Antioxid Redox Signal 2007; 9: 1115.

14. Zhang Q, Chang Q, Cox RA, Gong X, and Gould LJ: Hyperbaric oxygen attenuates apoptosis and decreases inflammation in an ischemic wound model. J Invest Dermatol 2008; 128: 2102.
15. Goldstein LJ, Gallagher KA, Bauer SM, Bauer RJ, Baireddy V, Liu ZJ, Buerk DG, Thom SR, and Velazquez OC: Endothelial progenitor cell release into circulation is triggered by hyperoxia-induced increases in bone marrow nitric oxide. Stem Cells 2006; 24: 2309

16. Londahl M, Katzman P, Nilsson A, and Hammarlund $\mathrm{C}$ : Hyperbaric oxygen therapy facilitates healing of chronic foot ulcers in patients with diabetes. Diabetes Care 2010; 33: 998.

17. Gordillo GM, Roy S, Khanna S, Schlanger R, Khandelwal S, Phillips G, and Sen CK: Topical oxygen therapy induces vascular endothelial growth factor expression and improves closure of clinically presented chronic wounds. Clin Exp Pharmacol Physiol 2008; 35: 957.

18. Kalliainen LK, Gordillo GM, Schlanger R, and Sen CK: Topical oxygen as an adjunct to wound healing: a clinical case series. Pathophysiology 2003; 9: 81.

19. Andrikopoulou E, Zhang X, Sebastian R, Marti G, Liu L, Milner SM, and Harmon JW: Current insights into the role of HIF-1 in cutaneous wound healing. Curr Mol Med 2011; 11: 218.

20. Zhou Z, Joslin S, Dellinger A, Ehrich M, Brooks B, Ren 0 , Rodeck U, Lenk R, and Kepley CL: A novel class of compounds with cutaneous wound healing properties. J Biomed Nanotechnol 2010; 6: 605 . 